\title{
POCT im Rettungsdienst: Was Medizintechnik über den Patienten verraten könnte
}

Jörg Johannes

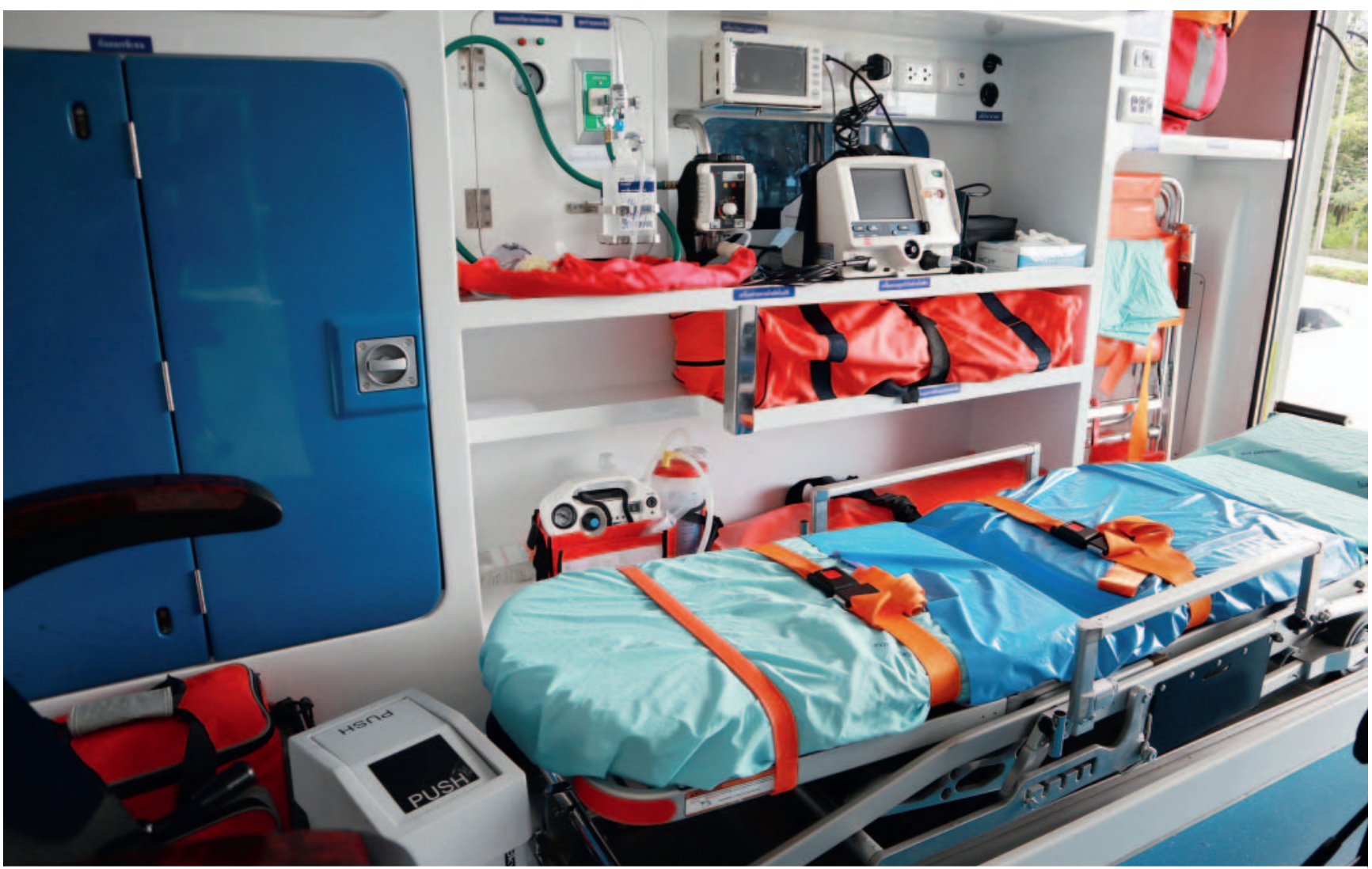

Quelle: nukies1234/stock.adobe.com

Dank des technischen Fortschritts wird das präklinische Monitoring stetig besser. Dabei hat sich der Umfang der erhobenen Parameter über Jahrzehnte kaum verändert. Gleichzeitig werden im Rettungsdienst vermehrt invasive Therapie- und Bridgingverfahren eingesetzt. Dies könnte zukünftig eine Ausweitung des präklinischen Standardmonitorings notwendig machen. Möglicherweise könnten dadurch sogar Ressourcen der Notfallversorgung entlastet werden.

\section{Was ist POCT?}

Point of Care Testing (POCT) beschreibt die dezentrale, patientennahe, bettseitige Labordiagnostik - also die Labordiagnostik direkt am Krankenbett. POCT-Systeme werden im stationären und im ambulanten Bereich eingesetzt. Auch Selbsttestgeräte für Patienten zählen zu den POCT-Systemen [1].

Derzeit sind die verfügbaren POCT-Verfahren überwiegend auf automatisch messbare Parameter begrenzt.
Dazu zählen z. B. die Parameter der Blutgasanalytik und die Bestimmung des Blutglukosespiegels, die schon lange bettseitig bestimmt werden. Zurzeit werden für mehr als 100 Einzelparameter POCT-Systeme angeboten.

Dank moderner technischer Entwicklungen stehen heute kleine („handheld“) Geräte zur Verfügung. Mit diesen lassen sich ein oder mehrere Parameter (nacheinander oder parallel) quantitativ bestimmen. Die benötigten chemischen Prüfmittel werden häufig fertig portioniert und vorbereitet als Unit-use-Reagenzien angeboten [2]. 
Merke

Die rechtlichen Grundlagen zum Point of Care Testing regelt, neben dem Medizinproduktegesetz, hauptsächlich die Richtlinie der Bundesärztekammer zur Qualitätssicherung labormedizinischer Untersuchungen (RiliBÄK) [3].

\section{Vorteile}

Die Geräte sind oft einfach bedienbar, und es ist keine Erfahrung oder Ausbildung in Labormedizin erforderlich. Der Probentransport in das Labor und ggf. die Probenvorbereitung entfallen. Der Bearbeitungszeitraum zwischen Indikationsstellung und der Verfügbarkeit der Ergebnisse - die Turn-around Time (TAT) - wird deutlich kürzer. Die somit rascher verfügbaren Testergebnisse können ggf. zu einer frühzeitigeren Entscheidung über die weitere Therapie oder Diagnostik führen [2]. Die durchschnittliche TAT bei POCT-Verfahren wird mit 5 bis 15 Minuten angegeben [4].

\section{Limitierende Faktoren}

Die Kosten für POCT-Messungen sind oft höher als diejenigen für entsprechende Untersuchungen im zentralen Labor. Dieser Vergleich ist aber nur für den innerklinischen Bereich interessant, da die Fixkosten für das zentrale Labor oft gleichzeitig bestehen bleiben [2]. Im Rettungsdienst würden die Anschaffung und der Einsatz von Geräten zur patientennahen Sofortdiagnostik vollkommen neue Kosten verursachen - die langfristig vermutlich eine Anpassung der Beförderungsentgelte notwendig machen.

Auch der erhebliche Schulungsbedarf des Personals muss berücksichtigt werden. Neben rechtlichen Aspekten geht es dabei vor allem um die sachgerechte Probengewinnung (Präanalytik) und die Vermeidung fehlerhafter Messungen und Interpretationen. Ein zentraler POCT-Koordinator kann daher sinnvoll sein. Dieses Vorgehen hat sich im innerklinischen Bereich bewährt [2].

In der präklinischen Notfallversorgung müssen auch das Platzangebot und mögliche Erschütterungen der Geräte berücksichtigt werden. Temperaturschwankungen können ebenfalls zum Problem werden, da die Geräte meist Raumtemperatur benötigen [5].

\section{Merke}

Der leichte Zugriff auf POC-Testverfahren könnte dazu verleiten, häufiger medizinisch nicht indizierte Analysen durchzuführen [4], was die Einsatzdauer und die Kosten ungerechtfertigt erhöhen könnte.

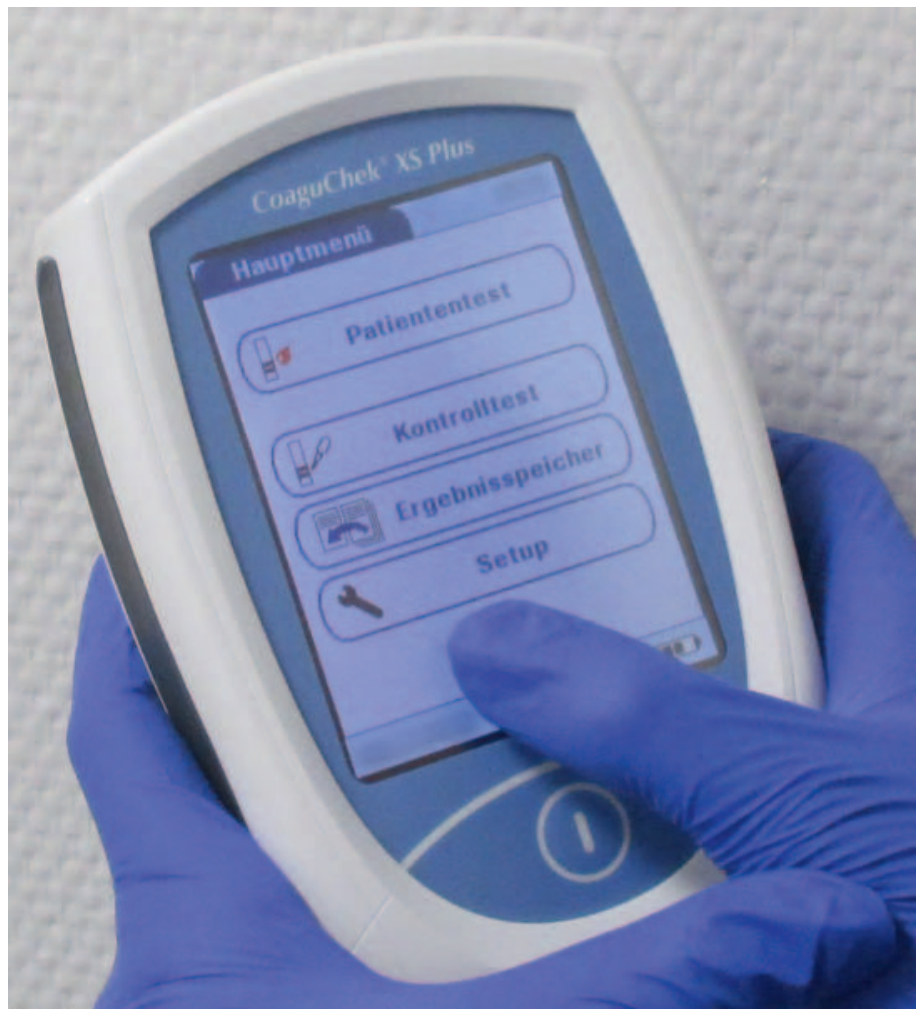

- Abb. 1 POCT-System im „Handheld“-Format.

\section{Einsatz im Rettungsdienst}

POCT-Verfahren machen es möglich, therapie- und diagnoserelevante Parameter frühzeitig zu bestimmen, wodurch ihre Bedeutung in der präklinischen Notfallversorgung weiter zunimmt [2, 6], (• Abb. 1).

Natürlich sind nur einige der patientennah bestimmbaren Parameter im Rettungsdienst relevant (

Merke

Hochsensitive Troponin-Tests ermöglichen die frühere Diagnose des Myokardinfarktes. Andere kardiale Biomarker (D-Dimere, NT-pro-BNP etc.) werden zur Abklärung von Differenzialdiagnosen benötigt [7].

Bei der Entscheidung, welche Parameter sinnvoll mittels patientennaher Sofortdiagnostik bestimmt werden können, sind auch Struktur und Lage des jeweiligen Rettungsdienstbereichs zu berücksichtigen: In einer ländlich geprägten Region mit langen Anfahrtszeiten und einer geringen Klinikdichte ist ein breiteres Parameterspektrum sinnvoller als in einer Stadt oder einem Ballungsgebiet. Dabei ist auch die tatsächliche Turn-around Time einzelner Messwerte und Geräte zu berücksichtigen ( $\triangleright$ Tab. 2) [8]. Diese kann im städtischen Bereich die Transportzeit in die Klinik überschreiten. 
- Tab. 1 POCT-Parameter mit möglicher Relevanz im Rettungsdienst [15].

\begin{tabular}{|l|l|}
\hline $\begin{array}{l}\text { Möglicher Einsatzbereich } \\
\text { im Rettungsdienst }\end{array}$ & Parameter \\
\hline Säure-Basen-Haushalt & $\mathrm{PH}, \mathrm{BE}, \mathrm{HCO}_{3}^{-}$ \\
\hline Blutgase & $\mathrm{CO}_{2}, \mathrm{O}_{2}$ \\
\hline Elektrolyte & $\mathrm{Na}^{+}, \mathrm{K}^{+}, \mathrm{Cl}^{-}$; ionisiertes $\mathrm{Ca}^{2+}$ \\
\hline Gerinnungsdiagnostik & $\mathrm{D}-$ Dimere, Quick, INR, ACT \\
\hline Hämatologie & Hämoglobin, Hämatokrit \\
\hline Diabetes mellitus & Clukose, Ketone \\
\hline kardiale Marker & $\begin{array}{l}\text { Troponin I, Troponin T, hochsensitives Troponin, } \\
\text { CK-MB, BNP, NT-pro-BNP }\end{array}$ \\
\hline Toxikologie & Alkohol, Medikamente, Drogen \\
\hline Entzündungsparameter & CRP, PCT \\
\hline Hämoglobinfraktionen & CO-Oxymetrie \\
\hline
\end{tabular}

- Tab. 2 Mögliche Messdauer kardialer Parameter am Beispiel des Cobas h 232 der Firma Roche [8].

\begin{tabular}{|l|l|l|l|}
\hline Kardialer Marker & Messbereich & $\begin{array}{l}\text { Unterstützte } \\
\text { Einheiten }\end{array}$ & Messdauer \\
\hline Troponin T & $40-2000 \mathrm{ng} / \mathrm{l}$ & $\begin{array}{l}\mathrm{ng} / \mathrm{l}, \mathrm{pg} / \mathrm{l}, \\
\mathrm{ng} / \mathrm{ml}, \mu \mathrm{g} / \mathrm{l}\end{array}$ & $12 \mathrm{~min}$ \\
\hline $\begin{array}{l}\text { Troponin T } \\
\text { Quantitative }\end{array}$ & $50-2000 \mathrm{ng} / \mathrm{l}^{1}$ & $\mathrm{ng} / \mathrm{l}$ & $12 \mathrm{~min}$ \\
\hline NT-proBNP & $60-9000 \mathrm{pg} / \mathrm{ml}$ & $\mathrm{pg} / \mathrm{ml}$ & $12 \mathrm{~min}$ \\
\hline D-Dimer & $0,1-4,0 \mu \mathrm{g} / \mathrm{ml}$ & $\mu \mathrm{\mu g} / \mathrm{ml}, \mathrm{ng} / \mathrm{ml}$, & $8 \mathrm{~min}$ \\
\hline Myoglobin & $30-700 \mathrm{ng} / \mathrm{ml}$ & $\mathrm{ng} / \mathrm{ml}$ & $\mathbf{m g} / \mathrm{min}$ \\
\hline CK-MB & $1,0-40 \mathrm{ng} / \mathrm{ml}$ & $\mathrm{ng} / \mathrm{ml}$ & $12 \mathrm{~min}$ \\
\hline 1 Der Bereich von $50-100 \mathrm{ng} / \mathrm{list}$ semiquantitativ. & \\
\hline
\end{tabular}

\section{Nur für Notärzte und Notärztinnen?}

Aus wirtschaftlicher Sicht scheint die Nutzung von POCTSystemen nur auf notärztlich besetzten Rettungsmitteln naheliegend. So wäre die benötigte Zahl an Geräten geringer. Die Interpretation vieler über POCT bestimmbarer Messwerte ist zurzeit nicht regelhaft Teil der Ausbildung von Notfallsanitätern/-innen. Dies könnte ebenfalls ein Argument dafür sein, den Einsatz von POCT-Geräten auf ärztlich besetzte Rettungsmittel zu begrenzen.

Bei der Analyse der ambulanten und präklinischen Notfallversorgung in Deutschland stellt man fest, dass beide Bereiche regelmäßig überlastet sind. Die rettungsdienstlichen Einsatzzahlen steigen ebenso kontinuierlich wie die Patientenzahlen in den Notaufnahmen und Rettungsstellen. Gleichzeitig nehmen Morbidität und Krank- heitsschwere weiter zu [9]. Auch bei den Kapazitäten der intensivmedizinischen Versorgung kommt es regelmäßig zu Engpässen [10].

Ob ein notärztlich besetztes Rettungsmittel nachgefordert wird, ob ein Intensivbett benötigt wird oder welche Fachabteilungen die Zielklinik aufweisen muss, wird meist anhand des Leitsymptoms und des klinischen Bilds entschieden. Da POCT-Systeme eine schnelle Abklärung bzw. einen Ausschluss relevanter Differenzialdiagnosen ermöglichen, scheint ihr Einsatz auch auf Rettungswagen sinnvoll. Neben der Schonung der notärztlichen Ressourcen ist eine differenziertere und zielgerichtetere Wahl der Zielklinik - insbesondere bezüglich der intensivmedizinischen Kapazitäten - wahrscheinlich.

\section{Merke \\ Die Fehlerquote bei der notärztlichen Einschätzung der Überwachungspflichtigkeit von Notfallpatienten/-innen kann bis zu $50 \%$ betragen. Dies führt mitunter zu einer nicht notwendigen Belastung von Intensivkapazitäten [11]. \\ POCT am Beispiel der arteriellen Blutgasanalyse}

Die arterielle Blutgasanalyse (BGA) ist ein unverzichtbarer Teil der klinischen Notfallversorgung. Sie detektiert schnell therapieentscheidende Werte, die noninvasive Messverfahren nicht erfassen. Neben den Blutgasen werden meist der Säure-Basen-Haushalt, die Elektrolyte, das Hämoglobin und der Blutzuckerwert bestimmt [5].

Im Intensivtransport ist der Einsatz von BGA-Geräten mittlerweile Routine. Dies zeigt, dass ein sicherer und zuverlässiger Einsatz im Rettungsdienst möglich ist. Trotzdem ist die BGA im Regelrettungsdienst weiterhin die Ausnahme, obwohl bereits seit 1990 Empfehlungen für deren präklinischen Einsatz vorliegen [5].

Untersuchungen lassen einen Überlebensvorteil für Patienten/-innen nach bestimmten Interventionen (z. B. Defibrillation) vermuten, wenn im Lauf der präklinischen Versorgung eine BGA durchgeführt wurde [12].

In einer anderen Untersuchung wurde bei kritisch Erkrankten oder Verletzten (NACA $\geq 5$ ) bereits am Notfallort mindestens eine arterielle BGA durchgeführt - von denen $97 \%$ mindestens einen pathologischen Wert zeigten. Bei $63 \%$ der Patienten/-innen erfolgte eine Intervention aufgrund eines pathologischen Werts in der BGA, der mit nichtinvasiven Verfahren nicht feststellbar gewesen wäre. Dies schließt Veränderungen der Respiratoreinstellungen bzw. die Indikationsstellung zur Beatmung ein $[5,13]$. Ob die präklinische Bestimmung venöser Blutgase ähnlich positive Ergebnisse erreicht, wurde bisher 
nicht untersucht. Diese könnte durch geschulte Notfallsanitäter/-innen bereits auf dem RTW erfolgen.

\section{Die Sache mit dem $\mathrm{CO}_{2}$}

Die normale Differenz zwischen dem exspiratorisch gemessenen $\mathrm{CO}_{2}\left(\right.$ etCO $\left.\mathrm{C}_{2}\right)$ und dem arteriellen $\mathrm{CO}_{2}$-Partialdruck $\left(\mathrm{paCO}_{2}\right)$ beträgt ca. $2-5 \mathrm{mmHg}\left(\mathrm{paCO}_{2}>\right.$ etCO $\left.\mathrm{CO}_{2}\right)$. Wie mehrere Studien zeigen, kann diese arterio-endexspiratorische $\mathrm{CO}_{2}$-Differenz bei Notfallpatienten/-innen deutlich zunehmen [14]. Es werden durchschnittliche Abweichungen von bis zu $17 \mathrm{mmHg}$ angegeben - mit einer Spannbreite von $-8,5$ bis $42,9 \mathrm{mmHg}$ [5]. Dabei kann nicht vorhergesagt werden, bei welchen Patienten/-innen mit einer hohen Abweichung zu rechnen ist [14]. Vor allem Verletzungen und Erkrankungen, die das Ventilations-Perfusions-Verhältnis (V/Q) verändern, scheinen die arterio-endexspiratorische $\mathrm{CO}_{2}$-Differenz zu erhöhen [5].

\section{Cave}

Die Messung des etCO ${ }_{2}$ scheint präklinisch bei kritisch Kranken oder Verletzten nicht ausreichend zuverlässig. Die Verwendung einer arteriellen POCT-Blutgasanalyse zur Respiratorsteuerung ist daher sinnvoll [5].

\section{KERNAUSSAGEN}

- Point of Care Testing ermöglicht die schnelle Labordiagnostik am Patientenbett und reduziert die Turn-around Time.

- Moderne Geräte und die Zunahme invasiver Therapien lassen den präklinischen Einsatz von POCT sinnvoll erscheinen. Dies gilt besonders für kardiale Marker und die arterielle BGA.

- Mögliche Vorteile einer venösen BGA bereits auf dem RTW müssen noch untersucht werden.

- Bei der Wahl des Systems und der Parameter sollten Struktur und Größe des Rettungsdienstbereichs berücksichtigt werden.

- Das Personal muss ausreichend im Umgang mit den Geräten, der Gewinnung der Proben (Präanalytik) und der Interpretation der Werte geschult sein.

- Das etCO ${ }_{2}$ ist zur Beatmungssteuerung bei kritisch Kranken oder Verletzten in der Präklinik nicht ausreichend zuverlässig.

- POCT im Rettungsdienst könnte notärztliche und innerklinische Ressourcen schonen.

\section{Interessenkonflikt}

Die Autoren geben an, dass kein Interessenkonflikt besteht.

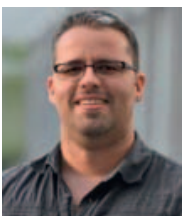

\section{Jörg Johannes}

Jahrgang 1981. 2001 Krankenpfleger. 2000 Rettungssanitäter. 2002 Rettungsassistent. 2013 Fachkrankenpfleger für Intensivpflege. 2016 Notfallsanitäter. Lehrrettungsassistent und Praxisanleiter im Gesundheitswesen. Derzeit berufsbegleitendes Studium Health Care Education/Gesundheitspädagogik. Seit Oktober 2018 an der Pflegeakademie am Klinikum Ludwigshafen am Rhein gGmbH für die Fachweiterbildung Notfallpflege verantwortlich.

Korrespondenzadresse

Jörg Johannes

Klinikum der Stadt Ludwigshafen am Rhein gGmbH Bremserstraße 79 in 67063 Ludwigshafen am Rhein Tel.: ++49/621/5032372 johannej@klilu.de

Literatur

[1] Lenzen-Schulte M. Point-of-Care-Diagnostik: Das Labor in der Kitteltasche. Dtsch Ärztebl 2016; 113: A1396

[2] Junker R, Schlebusch H, Luppa PB. Point-of-Care Testing in Hospitals and Primary Care. Dtsch Aerzteblatt Int 2010; 107: 561-567

[3] Bundesärztekammer. Richtlinie der Bundesärztekammer zur Qualitätssicherung laboratoriumsmedizinischer Untersuchungen. Gemäß dem Beschluss des Vorstands der Bundesärztekammer vom 11.04.2014 und 20.06.2014. Dtsch Ärztebl 2014; 111: A1583-A1618

[4] Schlüter B, Junker R. Labordiagnostik: Schneller ist nicht immer besser. Dtsch Ärztebl 2003; 100: A87-A89

[5] Hahn B. Erhöht invasives Monitoring die Patientensicherheit im Rettungsdienst? Eine prospektive Untersuchung zur prähospitalen invasiven Blutdruckmessung und Blutgasanalyse bei kritisch kranken und verletzten Patienten. Dissertation, Philipps-Universität Marburg, 2016. Im Internet: https:// archiv.ub.uni-marburg.de/diss/z2016/0762/pdf/dbh.pdf

[6] Wünning M. Point-of-Care-Testing in der Notfallmedizin. Manag Krankenh 2012. Im Internet: https://www.manage ment-krankenhaus.de/topstories/labor-diagnostik/pointcare-testing-der-notfallmedizin

[7] Achenbach S, Hamm C. Akutes Koronarsyndrom ohne ST-Hebung (NSTE-ACS). ESC Pocket Guidelines. Deutsche Gesellschaft für Kardiologie - Herz- und Kreislaufforschung 2012. Im Internet: https://leitlinien.dgk.org/files/2012_PocketLeitlinie_Akutes_Koronarsyndrom_NSTE-ACS.pdf

[8] Das cobas h 232 POC System - Unterstützte Streifen und Kontrollen. 2019. im Internet: https://www.roche.de/diagnos tics/systeme/point-of-care-diagnostik/cobas-h-232.html\# Uebersicht

[9] [Anonym]. Notaufnahmen und Rettungsdienste sind stark überlastet. Dtsch Aerzteblatt Online 2016. im Internet: https://www.aerzteblatt.de/nachrichten/71591/Notaufnah men-und-Rettungsdienste-sind-stark-ueberlastet

[10] Karagiannidis C, Kluge S, Riessen R et al. Auswirkungen des Pflegepersonalmangels auf die intensivmedizinische Versorgungskapazität in Deutschland. Med Klin - Intensivmed Notfallmedizin 2019; 114: 327-333 
[11] Bernhard M, Trautwein S, Stepan R et al. Notärztliche Einschätzung der klinischen Weiterversorgung von Notfallpatienten. Anaesthesist 2014; 63: 394-400

[12] Schatz M. Stellenwert von Laboruntersuchungen im Notarztdienst. 2008. Im Internet: https://www.google.de/url?sa= $\mathrm{t} \& \mathrm{rct}=\mathrm{j} \& \mathrm{q}=\&$ esrc $=\mathrm{s} \&$ source $=$ web $\& \mathrm{~cd}=1 \& \mathrm{cad}=\mathrm{rja} \& \mathrm{uact}=$ 8\&ved=2ahUKEwjtxdf32e7mAhWoPOwKHSL3BbgQFjAAeg QIAhAC\&url=https\%3A\%2F\%2Fonline.medunigraz.at\% 2Fmug_online\%2Fwbabs.getDocument\%3FpThesisNr\% 3D14721\%26pAutorNr\%3D59098\%26pOrgNR\% 3D1\&usg=AOvVaw1-62vhahJ2fe_0t7RPe2jP

[13] Hahn B, Wranze E, Wulf $\mathrm{H}$ et al. Mobiles Notfall-Labor in der präklinischen Notfallmedizin. Notarzt 2016; 32: 117-121
[14] Ensle G, Altemeyer KH. Arterio-endexspiratorische CO2-Differenz bei beatmeten Patienten in der Notfallmedizin. Notf Rettungsmedizin 1998; 1: 347-354

[15] Luppa P Hrsg. POCT - Patientennahe Labordiagnostik. 2. aktualisierte Aufl. Berlin: Springer Medizin; 2012

Bibliografie

DOI https://doi.org/10.1055/a-0964-1413

retten 2020: 9: 10-14

(c) Georg Thieme Verlag KG, Stuttgart · New York ISSN 2193-2387 